\title{
The combined effect of MC4R and FTO risk alleles on childhood obesity in Greece
}

\author{
Natalia Lazopoulou, ${ }^{1}$ Eleana Gioka, ${ }^{1}$ Ioanna Ntalla, ${ }^{2}$ Panagiota Pervanidou, ${ }^{1}$ \\ Alexandra-Maria Magiakou, ${ }^{1}$ Eleftheria Roma-Giannikou, ${ }^{1}$ George P. Chrousos, ${ }^{1}$ \\ Ioannis Papassotiriou, ${ }^{3}$ George Dedoussis, ${ }^{2}$ Christina Kanaka-Gantenbein ${ }^{1}$
}

${ }^{1}$ First Department of Paediatrics, University of Athens Medical School, "Aghia Sophia" Children's Hospital; ${ }^{2}$ Harokopio University of Dietetics; ${ }^{3}$ Department of Clinical Biochemistry, "Aghia Sophia" Children's Hospital; Athens, Greece

\begin{abstract}
OBJECTIVE: Single nucleotide polymorphism (SNP) risk alleles of obesogenic genes, such as the fat mass and obesity-associated protein (FTO) and the melanocortin-4 receptor (MC4R) gene, have been described in both paediatrics and adult populations. The aim of this study was to investigate the combined effect of FTO and MC4R risk alleles on the obese phenotype and metabolic profile of young children and adolescents of Greek origin. DESIGN: One hundred and fifty-three overweight and obese Greek children (mean age $11.08 \pm 2.23$ years) and 151 age-matched normal-weight controls were enrolled. Along with anthropometric and standard biochemical profile genetic analysis for the high-risk rs9939609 A allele of the FTO gene and the high-risk $C$ allele of the rs17782313 polymorphism downstream of the MC4R gene were investigated in all participants. RESULTS: The combined presence of three or more high-risk alleles of both the FTO and MC4R genes confers a 4-fold higher risk for obesity in children and adolescents of Greek origin, although these risk alleles have no impact on the metabolic alterations observed in these obese children and adolescents. CONCLUSIONS: There is a synergistic effect of the high-risk alleles of the FTO and MC4R genes on the obese phenotype, while no impact on the metabolic abnormalities was observed in Greek obese children and adolescents.
\end{abstract}

Key words: BMI, Childhood obesity, FTO, MC4R, Metabolic abnormalities, SNP

\section{INTRODUCTION}

Childhood obesity has evolved into a worldwide

Address for correspondence:

Christina Kanaka-Gantenbein, MD, PhD,

Associate Professor for Paediatrics Endocrinology-Juvenile

Diabetes, First Department of Paediatrics, University of Athens

Medical School, "Agia Sophia” Children's Hospital, Thivon

\& Livadias Str., GR 11527, Goudi, Athens, Greece, E-mail:

chriskan@med.uoa.gr

Received: 02-03-2014, Accepted: 18-06-2014 epidemic. It has been estimated that in 2010, 43 million children aged $<5$ years were overweight or obese and that by 2020 this percentage will have risen to 60 million children. ${ }^{1}$ Contrary to prior common beliefs, it has been proved that "heavy" children will grow into unhealthy, obese adults with a plethora of medical conditions, this rendering the current epidemic a global health crisis on multiple levels. Greece has the highest percentage of overweight and obese adolescents in Europe, at $44 \%$ and $11 \%$, respectively. ${ }^{2}$ 
The radical changes of lifestyle during the past 50 years have undoubtedly created an "obesogenic" environment characterized by sedentary habits and energy-dense palatable foods that are accountable for the present explosion in obesity. However, not everyone becomes overweight. Studies on heritability of human adiposity have estimated that genetic factors contribute up to $40-70 \%$ of the variance of adiposity. ${ }^{3,4}$ Genome Wide Association Studies (GWAS) have contributed to researching genetic variations of multiple loci. Still, in the case of common obesity single nucleotide polymorphisms (SNPs) variations can explain only up to $1.5 \%$ of the variance in BMI. ${ }^{4}$

The first "obesogenic" SNP to be replicated and validated was the fat mass and obesity-associated (FTO) gene consisting of nine exons on chromosome 16 (16q12.2). ${ }^{5}$ It encodes a 2-oxoglutarate-dependent nucleic acid demethylase ${ }^{6}$ and is found in higher concentrations in the hypothalamus. Its concentration in the arcuate nucleus fluctuates depending on the fasting state. Even though its physiological function has not yet been elucidated, evidence so far associates FTO variants with hyperphagia of calorie-dense foods. ${ }^{6,7}$ Recent studies reveal a strong correlation between the rs9939609 A allele of the FTO and childhood obesity. ${ }^{8,9}$ In this SNP, located in the first intron, threonine (T) is substituted by alanine (A) (T->A), this leading to a possible protein dysfunction that ultimately results in increased body weight.

Additionally, in humans gain-of-function mutations of the MC4R gene have been described as a relatively frequent genetic finding in cases of obesity. ${ }^{10-12}$ At least 72 polymorphisms of the MC4R gene have been described so far with a small number of them leading to formation of a defective, mutated MC4R. Among these mutations some seem to cause intracellular retention of the deformed protein, while others cause abnormal ligand binding or ligand/receptor defects. ${ }^{12-15}$ The common SNP rs 17782313 substituting a threonine amino acid (T) for cysteine (C) (T->C), lying $188 \mathrm{~kb}$ downstream of the MC4R gene, has recently been associated with increased body mass index (BMI) during childhood, affecting energy intake as well as energy expenditure, while in adults it has been associated with insulin resistance and diabetes risk. ${ }^{16-22}$

\section{Aim of the study}

The aim of the study was to investigate the impact of the high-risk $\mathrm{C}$ allele of the rs17782313 polymorphism of the MC4R gene and of the highrisk A allele of the rs 9939609 polymorphism of the FTO gene and their combined effect on the BMI and metabolic traits of overweight and obese children and adolescents in Greece.

\section{MATERIALS AND METHODS}

\section{Subjects and Methods}

\section{Overweight and obese subjects}

One hundred and fifty-three (153) obese Greek children examined in the Obesity Outpatient Clinic of the Division of Endocrinology, Diabetes and Metabolism of the First Dept. of Paediatrics of the University of Athens in "Aghia Sofia" Children's Hospital from 2006 to 2009 who were willing to participate were included in the study. Subjects were classified as overweight, obese and normal-weight according to the International Obesity Task Force (IOTF) cut-off limits. ${ }^{23}$ In order to avoid a probable heterogeneity in the allele frequencies between Greek and non-Greek Caucasians, only children of Greek origin were included in the present study. Exclusion criteria were underlying chronic illness, chronic medication and presence of a genetic syndrome, chromosomal disorders, mental disorders or pre-existing psychopathology.

\section{Normal-weight subjects (controls)}

Normal-weight children included in this study as controls were recruited for the GENDAI study. ${ }^{24}$ Briefly, normal-weight children were recruited from fifth and sixth grade classes of several elementary schools in the region of Attica, Greece. The selected schools (40 schools in a total of 593 in the region) were all in socioeconomically similar urban ureas. The controls were age-matched with the overweight/ obese group. The study was approved by the Ethical Committee of "Aghia Sofia" Children's Hospital and subjects were enrolled in the study only after informed written consent had been obtained from their parents or guardians.

\section{Anthropometric data}

Anthropometric measurements were obtained 
using classical methods. Briefly, all children were dressed in light clothes. For height measurement they were placed in the Frankfurt plane. Height was measured in centimeters and weight in kilograms. Waist circumference was measured using a soft tape around the $12^{\text {th }}$ rib, hip circumference around the iliac crest and the waist-to-hip ratio was calculated dividing the two measurements. BMI was calculated as the ratio of weight $/ \mathrm{height}^{2}\left(\mathrm{~kg} / \mathrm{m}^{2}\right)$. Classification of children as normal-weight, overweight and obese met the criteria defined by the IOTF. ${ }^{23}$

\section{Blood chemistry}

Blood was collected after an overnight fast at 8:00 a.m. The fasting glucose (Glu), total cholesterol (CHOL), triglycerides (TG), high-density lipoprotein cholesterol (HDL-C) and low-density lipoprotein cholesterol (LDL-C) concentrations in serum were assayed using the ADVIA 1800 Clinical Chemistry Analyzer (Siemens Healthcare Diagnostics, Tarrytown, NY, USA), while insulin levels were determined with an electrochemiluminescence immunoassay on the Roche ELECSYS 2010 immunoassay analyzer (Basel, $\mathrm{CH}$ ).

The HOMA insulin resistance (HOMA-IR) index was calculated according to the formula: HOMA-IR Index $=[$ (Fasting Insulin $) \times($ Fasting Glucose $)] / 161$, with insulin concentration expressed in pmoles/ 1 and glucose in mmoles/l.

\section{Genotyping SNPs rs 17782313 of MC4R and rs9939609 of FTO}

For the genetic analysis, DNA was extracted from white blood cells from whole blood sampling using standard methods. Single nucleotide polymorphisms rs17782313 and rs9939609 of the MC4R and FTO genes, respectively, were analyzed after Real Time PCR amplification using the LightCycler 2.0 (Roche, Mannheim, Germany). The Real Time PCR LightCycler assay is based on hybridization probes labeled with fluorescent dyes that allow fluorescence resonance energy transfer. ${ }^{25}$ SNP genotyping was carried out using melting curve analysis. Sequences of primers and probes used for Real Time PCR with the LightCycler 2.0 are available upon request.

\section{Statistical analysis}

Statistical analyses were performed using SPSS
20.0 for Windows (SPSS Inc., Chicago, IL, USA). Association statistics were calculated using PLINK software. ${ }^{26}$ Continuous variables are presented as mean values \pm standard deviation. The statistical test used to delineate statistical significance ( $p$-value) in normally distributed continuous variables between groups was the analysis of variance (ANOVA) test, as depicted in Table 1. For non-normally distributed variables, the natural logarithmical transformed values were used. Linear regression was performed to test the hypothesis of association between polymorphisms as predictors and their effect on anthropometric indices after adjustment for potential confounders (age, gender) (Table 2). Logistic regression was performed to determine the independent effect of risk alleles of MC4R and FTO on the relative likelihood of obesity.

Table 1. Descriptive characteristics of normal weight and overweight/ obese subjects

\begin{tabular}{|c|c|c|c|}
\hline & $\begin{array}{c}\text { Normal } \\
\text { weight }\end{array}$ & $\begin{array}{c}\text { Overweight/ } \\
\text { Obese }\end{array}$ & p-value \\
\hline Number & 151 & 153 & \\
\hline Age & $10.96 \pm 0.57$ & $11.08 \pm 2.23$ & 0.494 \\
\hline Weight (kg) & $37.96 \pm 5.7$ & $66.43 \pm 19.6$ & $<0.001$ \\
\hline Weight Z-score & $-0.48 \pm 0.78$ & $4.2 \pm 2.77$ & $<0.001$ \\
\hline Height (m) & $1.46 \pm 0.70$ & $1.49 \pm 0.12$ & 0.003 \\
\hline Height Z-score & $-0.12 \pm 0.98$ & $0.56 \pm 1.46$ & $<0.001$ \\
\hline BMI $\left(\mathrm{kg} / \mathrm{m}^{2}\right)$ & $17.75 \pm 1.5$ & $29.27 \pm 5.6$ & $<0.001$ \\
\hline BMI Z-score & $-0.44 \pm 0.44$ & $2.95 \pm 1.65$ & $<0.001$ \\
\hline $\begin{array}{l}\text { Waist circumference } \\
(\mathrm{cm})\end{array}$ & $61.99 \pm 5.23$ & $88.73 \pm 14.64$ & $<0.001$ \\
\hline Waist-Hip ratio & $0.77 \pm 0.04$ & $0.89 \pm 0.09$ & $<0.001$ \\
\hline Waist-Height ratio & $0.42 \pm 0.032$ & $0.59 \pm 0.082$ & 0.000 \\
\hline Birth weight (g) & $3202 \pm 459$ & $3366 \pm 479$ & 0.006 \\
\hline $\begin{array}{l}\text { Systolic blood } \\
\text { pressure (mm Hg) }\end{array}$ & $118 \pm 12.16$ & $116 \pm 16.8$ & 0.420 \\
\hline $\begin{array}{l}\text { Diastolic blood } \\
\text { pressure (mm Hg) }\end{array}$ & $74.02 \pm 10.8$ & $75.83 \pm 10.82$ & 0.234 \\
\hline Insulin (uU/ml) & $6.54 \pm 3.51$ & $14.46 \pm 6.77$ & $<0.001$ \\
\hline Glucose (mmol/1) & $4.70 \pm 0.38$ & $4.70 \pm 0.41$ & 0.935 \\
\hline HOMA-IR & $1.38 \pm 0.79$ & $3.06 \pm 1.49$ & $<0.001$ \\
\hline Cholesterol (mg/dl) & $186 \pm 27$ & $170 \pm 33$ & $<0.001$ \\
\hline HDL-C (mg/dl) & $54 \pm 9.7$ & $46 \pm 10$ & $<0.001$ \\
\hline LDL-C (mg/dl) & $120 \pm 22$ & $105 \pm 27$ & $<0.001$ \\
\hline Triglycerides (mg/dl) & $59 \pm 18$ & $79 \pm 31$ & $<0.001$ \\
\hline
\end{tabular}


Table 2. Linear regression analysis of the impact of the MC4R rs17782313 C allele and the FTO rs9939609 A allele on anthropometric indices and metabolic traits in all subjects

\begin{tabular}{lccccccc}
\hline & \multicolumn{2}{c}{ MC4R rs17782313 C allele } & & \multicolumn{3}{c}{ FTO rs9939609 A allele } \\
\cline { 2 - 3 } \cline { 6 - 7 } & Mean & SE & P-value & & Mean & SE & P-value \\
\hline Weight & 3.998 & 1.809 & 0.0279 & & 3.603 & 1.418 & 0.011 \\
Weight Z-score & 0.7053 & 0.3041 & 0.0210 & & 0.6168 & 0.2387 & 0.01 \\
Height & -0.0009 & 0.0073 & 0.8984 & & 0.0117 & 0.0057 & 0.04 \\
Height Z-score & 0.0503 & 0.1235 & 0.6842 & & 0.1828 & 0.0965 & 0.059 \\
BMI & 1.737 & 0.688 & 0.0122 & & 1.346 & 0.5426 & 0.013 \\
BMI Z-score & 0.5066 & 0.2063 & 0.0147 & & 0.3813 & 0.1629 & 0.02 \\
Waist circumference & 2.821 & 1.719 & 0.102 & & 3.58 & 1.391 & 0.01 \\
Waist-Hip ratio & 0.0094 & 0.0091 & 0.3033 & & 0.0179 & 0.0074 & 0.016 \\
Waist-Height ratio & 0.0189 & 0.0107 & 0.0779 & & 0.0201 & 0.0087 & 0.021 \\
Birth weight & -0.357 & 50.63 & 0.9944 & & 5.26 & 41 & 0.895 \\
Head circumference & 2.243 & 1.334 & 0.094 & & 2.227 & 1.086 & 0.041 \\
SBP & -1.921 & 1.742 & 0.2714 & & 2.186 & 1.405 & 0.121 \\
DBP & 0.355 & 1.12 & 0.9766 & & 0.0232 & 0.989 & 0.9813 \\
INS & 0.859 & 0.673 & 0.2029 & & 0.4711 & 0.5439 & 0.39 \\
INS (LN) & 0.0702 & 0.068 & 0.3033 & & 0.05071 & 0.0555 & 0.36 \\
GLC (MMOL) & 0.031 & 0.0405 & 0.4469 & & 0.0003 & 0.0326 & 0.9927 \\
HOMA IR & 0.201 & 0.1472 & 0.1743 & & 0.1137 & 0.1196 & 0.342 \\
HOMA (LN) & 0.0772 & 0.0706 & 0.2747 & & 0.05417 & 0.0578 & 0.349 \\
CHOL & 4.305 & 3.211 & 0.1812 & & -0.1612 & 2.557 & 0.949 \\
HDL-C & 1.168 & 1.084 & 0.282 & & -0.811 & 0.872 & 0.353 \\
LDL-C & 2.091 & 2.714 & 0.4416 & & -1.071 & 2.169 & 0.622 \\
TGS & 1.347 & 2.764 & 0.6264 & & 0.9453 & 2.217 & 0.670 \\
TGS (LN) & 0.0034 & 0.0375 & 0.928 & & 0.0185 & 0.03 & 0.539 \\
\hline
\end{tabular}

*BMI, BMI z-score and weight were adjusted for age and gender; birth weight and birth weight z-score were adjusted for gender; waist circumference and waist-hip ratio were adjusted for age, gender and BMI; glucose, insulin, HOMA-IR, cholesterol, HDL-cholesterol, LDL-cholesterol and triglycerides were adjusted for age, gender and BMI. ( ${ }^{\#}$ Mean \pm SD).

Logistic regression was also carried out to investigate the combined effect of the abovementioned risk alleles on the risk of becoming obese, as defined by Cole. ${ }^{23}$ In order to investigate the combined effects of the risk alleles on the relative likelihood of obesity among the children participating in the study, the study sample was stratified into three groups: the children whose genotypes contained no risk alleles of the examined genes (reference group), the children whose genotypes contained 1-2 risk alleles and the children with genotypes of 3-4 risk alleles of MC4R and FTO. These genotypes were considered as the predictor variables in our logistic regression model, while the risk of becoming obese was considered our dependent variable. The level of significance was set at $\mathrm{p}<0.05$. Odds ratios (ORs) with $95 \%$ confidence intervals $(95 \% \mathrm{CI})$ were calculated.

\section{RESULTS}

The descriptive characteristics for both groups included are presented in Table 1. The mean age was approximately 11 years for both groups $(\mathrm{p}>0.05)$ and the overweight/obese population had, as expected, an elevated BMI, BMI-z-score, weight and weight $\mathrm{z}$-score, waist circumference, waist-to-hip ratio, waist-to-height-ratio $(\mathrm{p}<0.001)$. The overweight/obese population also had a high birth weight $(\mathrm{p}=0.006)$ and increased height $\mathrm{z}$-score $(\mathrm{p}<0.001)$. 
Regarding the metabolic parameters of the two groups, the overweight/obese participants had significantly increased plasma insulin and HOMA-IR, higher serum triglyceride levels and a lower serum HDL-Cholesterol $(\mathrm{p}<0.001)$, while their serum glucose levels, systolic and diastolic blood pressure did not differ from the controls.

\section{MC4R polymorphisms and anthropometric and metabolic profile}

The observed genotype frequencies for the rs 17782313 polymorphism of the MC4R gene were in Hardy-Weinberg equilibrium for both normal-weight and obese participants ( $\mathrm{p}$-value $>0.05$ ). The minor allele frequency was 0.227 for all subjects: 0.188 and 0.256 for normal-weight and overweight/obese subjects, respectively (data not shown).

Associations of MC4R rs17782313 genotypes with basic anthropometric indices are shown in Table 2. The rs $17782313 \mathrm{C}$ risk allele schowed a significant association with BMI ( $p$-value $=0.01)$. Since there was no association to the height $(\mathrm{p}=0.89)$ the significant association to the BMI was through its impact on body weight $(p=0.029)$. We also attempted to replicate the associations of the SNP MC4R rs17782313 with the risk of being obese (as defined for children and adolescents according to $\mathrm{Cole}^{23}$ ). The relative likelihood of obesity in children with the high-risk allele of rs17782313 SNP was 1.5 times higher than in normal weight subjects ( $\mathrm{p}$-value $=0.03$ ).

Associations of the MC4R rs17782313 genotype with birth weight and markers of central adiposity (waist circumference and waist-hip ratio) were also investigated. However, no significant association was observed between the rs17782313 genotype and the birth weight or the indices of central adiposity. We further investigated whether the MC4R rs177823123 genotype was associated with metabolic parameters such as glucose or lipid metabolism indices; however, no significant association was observed.

\section{FTO polymorphisms and anthropometric and metabolic profile}

The observed genotype frequencies for rs9939609 of the FTO gene were in Hardy-Weinberg equilibrium for both normal-weight and obese subjects ( $\mathrm{p}$-value $>0.05$ ). The minor allele frequency was 0.440 for all subjects: 0.401 and 0.479 for normal-weight and obese subjects, respectively (data not shown).

Associations of FTO rs9939609 genotypes with basic anthropometric indices are shown in Table 2. The rs9939609 A risk allele showed significant association with BMI ( $\mathrm{p}$-value $=0.013$ ), this effect apparently coming about through its impact on body weight ( $p$-value $=0.011)$. Interestingly, in the case of FTO a significant association with height is observed $(p$-value $=0.040)$. We also attempted to replicate the association of the FTO SNP with the risk of being obese (as defined for children and adolescents according to Cole et $\mathrm{al}^{23}$ ). The relative likelihood of obesity in children with the high risk allele of the rs9939609 SNP was 1.4 times higher than for normal weight (p-value $=0.047)$.

As in the case of MC4R, associations of the FTO rs9939609 genotype with birth weight, markers of central adiposity (waist circumference and waist-hip ratio), glucose metabolism indices (glucose, insulin and HOMA-IR) and serum lipids (total cholesterol, HDL-cholesterol, LDL-cholesterol, triglycerides) were also tested. Waist circumference, waist to height and waist-to-hip ratios were all increased in children with the FTO risk allele (p-value $=0.01,0.02$ and 0.016, respectively) (Table 2). However, no association was found between the polymorphism and either glucose or lipid metabolism abnormalities.

\section{Combined effect of the high risk alleles on the obese phenotype}

We also applied logistic regression analysis in order to study the impact of combined risk alleles on the formation of an obese phenotype. Although the odds ratio of becoming obese was slightly elevated among children having one or two risk alleles (OR $1.059,95 \%$ CI $0.587-1.910, \mathrm{p}=0.85$ ), for those bearing three or four risk alleles, the OR was more than four times increased when compared with the control group (OR 4.471, 95\% CI 1.750 - $11.424 \mathrm{p}=0.002$ ), indicating a synergistic effect.

\section{DISCUSSION}

In the present study we investigated the impact of the presence of the risk alleles of two polymorphisms, one near the MC4R gene (rs17782313) that replaces 
a threonine amino acid with cysteine ( $\mathrm{T}->\mathrm{C})$ and one intrinsic to the FTO gene (rs9939609) that replaces a threonine amino acid with alanine $(\mathrm{T}->\mathrm{A})$ and their combined effect on childhood obesity. Both of them were significantly associated with the obese phenotype in Greek children and adolescents. Furthermore, both SNP risk alleles appear to affect weight and weight z-score $(p<0.05)$, implying that their effect on BMI is mainly through gaining weight. The combined presence of three or more high risk alleles of both the FTO and MC4R genes confers a 4-fold higher risk for obesity in children and adolescents of Greek origin. Therefore, the combined effect of FTO and MC4R risk alleles was positively associated with an even higher risk for obesity, which is in accordance with findings of larger studies both in children and

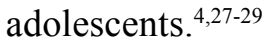

A common finding of large-scale studies is that genetic variations of MC4R and FTO have their maximum effect during childhood that improves during adult life. ${ }^{17,22,30,31}$ Elks et al in a recent longitudinal study found a positive effect of FTO and MC4R risk alleles on adiposity traits (BMI, weight) but also a positive effect on height during preadolescence, that attenuates with age. ${ }^{27}$ The authors suggest that the age of 11 years, which is the mean age of our sample, is a landmark for development and that obesity affects growth tempo, making overweight/obese children taller up to pre-adolescence. They further report that during the transition from adolescence to early adulthood adiposity no longer contributes to a taller stature. ${ }^{27}$ Sovio et al ${ }^{22}$ have reported a positive association between the rs 9939609 A allele and BMI after the age of 5.5 years. They also report that this particular SNP accelerates the developmental age by $2.4 \%$ per risk allele, corresponding to an early adiposity rebound and eventually early puberty. ${ }^{32}$ Haworth et al state that the rs9939609 A polymorphism progressively becomes more strongly associated with BMI after the age of $7 .{ }^{33}$ Specifically in the case of twins, they report that heritability for BMI increased from $48 \%$ at the age of 4 years to $78 \%$ at the age of 11 years. Their results are consistent with the findings of previous studies on the effects of adiposity on the childhood growth trajectories. ${ }^{34}$ Contrary to results presented by Elks et al, the rs9939609 A allele had no effect on height in our cohort. In the present study we observed that the FTO risk allele was associated with height in the obese population $(\mathrm{p}=0.040)$, and this effect was attenuated after adjustment for age and $\operatorname{sex}(\mathrm{p}=0.059)$, maintaining a positive trend that replicates the theory that this is the age when genetic predisposition typically affects growth.

Moreover, even though our obese group had increased birth weight $(\mathrm{p}=0.006)$, there was no correlation of this index to either the FTO or the MC4R risk alleles. This finding is in accordance with other studies. ${ }^{17,30,32,35,36}$ Elks et al report a marginal positive correlation of birth weight to the obesity-risk-allele-score, but the correlation was significantly more robust after the first 6 weeks of life. ${ }^{37}$ The FTO and MC4R risk alleles are thought to contribute to BMI increase through regulation of central nervous system messages to the hypothalamus, regulating appetite, satiety and calorie-dense preferences. Birth weight is strongly influenced by exogenous, mainly maternal, choices in the quantity and quality of provided energy, an effect that partly explains why the genetic risk alleles that modulate eating behavior have a minimal effect on weight. It could also imply that our obese population was born in a strongly "obesogenic" environment that has affected their prenatal development.

No association was found between the FTO and/or MC4R risk alleles and metabolic traits in our population. However, the obese/overweight subgroup already had insulin resistance and a dyslipidemic profile (low HDL-cholesterol and higher serum triglyceride levels), findings that are strongly associated with adiposity in both adults and children. ${ }^{38}$ Our obese subpopulation did not exhibit high blood pressure, although previously, Xi Bo et al reported a positive association of the FTO risk allele with hypertension among obese homozygotes. ${ }^{29}$ Their population extended to 18 years of age though, whereas our population is younger and has possibly been exposed for less time to the atherogenic effects of adiposity.

In conclusion, we found that the high risk alleles rs17782313 SNP of the MC4R gene and rs9939609 of the FTO gene had a significant impact on the occurrence of the obese phenotype in Greek children and adolescents. We did not, however, observe a 
direct association between these high-risk alleles and the metabolic profile of the enrolled children, even though our obese subgroup already presented anthropometric and metabolic traits of central adiposity (waist-to-hip ratio, insulin resistance, low HDL, increased triglyceride levels).

The present study in the paediatric Greek population confirms the findings of similar large-scale studies conducted in other populations. It seems that the FTO and MC4R genes affect BMI and weight through modifications in eating behavior and habits, appetite and satiety, and not via alterations in energy expenditure. Pre-adolescence is the best time to study genetic influences, because eating behavior becomes less influenced by the family environment as the individual grows and forms his/her own tastes and habits. In early childhood, the youngster is highly family-dependent concerning the quality, quantity and schedule of meals, while during the transition to early adulthood eating habits are largely determined by social, cultural and psychological influences. ${ }^{39}$ Earlier studies have demonstrated that lifestyle, physical activity and/or sedentary habits influence the expression of FTO and MC4R both in children and in adults. ${ }^{40-42}$

As the need for new treatment strategies to combat obesity becomes mandatory, more studies need to be carried out to elucidate the role of the MC4R and FTO polymorphisms in obesity and to determine whether they are associated with the development of lipid and glucose abnormalities as these children grow into adults. The obesity risk alleles have additive effects on the occurrence of the obese phenotype, but they can also be influenced and attenuated by lifestyle modification. This is why proper genetic screening of susceptible individuals at a preschool age may contribute to the establishment of healthy lifestyle habits, thereby alleviating the deleterious effects of persisting adiposity.

\section{CONFLICTS OF INTEREST}

The authors report no conflict of interest.

\section{FUNDING}

None for all authors.

\section{REFERENCES}

1. Dietz WH, 1998 Health consequences of obesity in youth: childhood predictors of adult disease. Pediatrics 101: 518-525.

2. Brug J, van Stralen MM, te Velde SJ, et al, 2012 Differences in weight status and energy-balance related behaviors among schoolchildren across Europe: The ENERGY-Project. PLoS ONE 7: e34742.

3. Chung WK, Leibel RL, 2008 Considerations regarding the genetics of obesity. Obesity (Silver Spring) 16: Suppl 3: 33-39.

4. Speliotes EK, Willer CJ, Berndt SI, et al, 2010 Association analyses of 249,796 individuals reveal 18 new loci associated with body mass index. Nat Genet 42 : 937-948.

5. Frayling TM, Timpson NJ, Weedon MN, et al, 2007 A common variant in the FTO gene is associated with body mass index and predisposes to childhood and adult obesity. Science (New York, NY) 316: 889-894.

6. Wardle J, Carnell S, Haworth CMA, Farooqi IS, O'Rahilly S, Plomin R, 2008 Obesity associated genetic variation in FTO Is associated with diminished satiety. J Clin Endocrinol Metab 93: 3640-3643.

7. Tanofsky-Kraff M, Han JC, Anandalingam K, et al, 2009 The FTO gene rs9939609 obesity-risk allele and loss of control over eating. Am J Clin Nutr 90: 1483-1488.

8. Xi B, Zhang M, Wang C, et al, 2013 The common SNP (rs9939609) in the FTO gene modifies the association between obesity and high blood pressure in Chinese children. Mol Biol Rep 40: 773-778.

9. Rendo T, Moleres A, Marti Del Moral A, 2009 Effects of the FTO gene on lifestyle intervention studies in children. Obes Facts 2: 393-399.

10. Vaisse C, Clement K, Guy-Grand B, Froguel P, 1998 A frameshift mutation in human MC4R is associated with a dominant form of obesity. Nat Genet 20: 113-114.

11. Vaisse C, Clement K, Durand E, Hercberg S, GuyGrand B, Froguel P, 2000 Melanocortin-4 receptor mutations are a frequent and heterogeneous cause of morbid obesity. J Clin Invest 106: 253-262.

12. Yeo GS, Lank EJ, Farooqi IS, Keogh J, Challis BG, O'Rahilly S, 2003 Mutations in the human melanocortin-4 receptor gene associated with severe familial obesity disrupts receptor function through multiple molecular mechanisms. Hum Mol Genet 12: 561-574.

13. Lubrano-Berthelier C, Cavazos M, Le Stunff C, et al, 2003 The human MC4R promoter: characterization and role in obesity. Diabetes 52: 2996-3000.

14. Lubrano-Berthelier C, Durand E, Dubern B, et al, 2003 Intracellular retention is a common characteristic of childhood obesity-associated MC4R mutations. Hum Mol Genet 12: 145-153.

15. Nijenhuis WA, Garner KM, van Rozen RJ, Adan RA, 2003 Poor cell surface expression of human melanocortin-4 receptor mutations associated with obesity. $\mathrm{J}$ 
Biol Chem 278: 22939-22945.

16. Qi L, Kraft P, Hunter DJ, Hu FB, 2008 The common obesity variant near MC4R gene is associated with higher intakes of total energy and dietary fat, weight change and diabetes risk in women. Hum Mol Genet 17: 3502-3508.

17. Loos RJF, Lindgren CM, Li S, et al, 2008 Common variants near MC4R are associated with fat mass, weight and risk of obesity. Nat Genet 40: 768-775.

18. Cauchi S, Stutzmann F, Cavalcanti-Proença C, et al, 2009 Combined effects of MC4R and FTO common genetic variants on obesity in European general populations. J Mol Med 87: 537-546.

19. Kring SI, Holst C, Toubro S, et al, 2010 Common variants near MC4R in relation to body fat, body fat distribution, metabolic traits and energy expenditure. Int J Obes (Lond) 34: 182-189.

20. Liu G, Zhu H, Lagou V, et al, 2010 Common variants near melanocortin 4 receptor are associated with general and visceral adiposity in European- and AfricanAmerican youth. J Pediatr 156: 598-605e1.

21. Grant SF, Bradfield JP, Zhang H, et al, 2009 Investigation of the locus near MC4R with childhood obesity in Americans of European and African ancestry. Obesity (Silver Spring) 17: 1461-1465.

22. Hardy R, Wills AK, Wong A, et al, 2010 Life course variations in the associations between FTO and MC4R gene variants and body size. Hum Mol Genet 19: 545 552.

23. Cole TJ, Bellizzi MC, Flegal KM, Dietz WH, 2000 Establishing a standard definition for child overweight and obesity worldwide: international survey. BMJ 320: 1240-1243.

24. Papoutsakis C, Vidra NV, Hatzopoulou I, et al, 2007 The gene-diet Attica investigation on childhood obesity (GENDAI): overview of the study design. Clin Chem Lab Med 45: 309-315.

25. Blomeke B, Sieben S, Spotter D, Landt O, Merk HF, 1999 Identification of $\mathrm{N}$-acetyltransferase 2 genotypes by continuous monitoring of fluorogenic hybridization probes. Anal Biochem 275: 93-97.

26. Purcell S, Neale B, Todd-Brown K, et al, 2007 PLINK: a tool set for whole-genome association and populationbased linkage analyses. Am J Hum Genet 81: 559-575.

27. Elks CE, Loos RJF, Hardy R, et al, 2012 Adult obesity susceptibility variants are associated with greater childhood weight gain and a faster tempo of growth: the 1946 British Birth Cohort Study123. Am J Clin Nutr 95: 1150-1156.

28. Graff M, North KE, Mohlke KL, et al, 2012 Estimation of genetic effects on BMI during adolescence in an ethnically diverse cohort: The National Longitudinal Study of Adolescent Health. Nutr Diabetes 2: e47.

29. Xi B, Zhang M, Wang C, et al, 2013 The common SNP (rs9939609) in the FTO gene modifies the association between obesity and high blood pressure in Chinese children. Mol Biol Rep 40: 773-778.

30. Wu L, Xi B, Zhang M, et al, 2010 Associations of six single nucleotide polymorphisms in obesity-related genes with BMI and risk of obesity in Chinese children. Diabetes 59: 3085-3089.

31. Xi B, Chandak GR, Shen Y, Wang Q, Zhou D, 2012 Association between common polymorphism near the MC4R gene and obesity risk: a systematic review and meta-analysis. PLoS ONE 7: e45731.

32. Sovio U, Mook-Kanamori DO, Warrington NM, et al, 2011 Association between common variation at the FTO locus and changes in body mass index from infancy to late childhood: the complex nature of genetic association through growth and development. PLoS Genet 7: e1001307-e07.

33. Haworth CM, Carnell S, Meaburn EL, Davis OS, Plomin R, Wardle J, 2008 Increasing heritability of $\mathrm{BMI}$ and stronger associations with the FTO gene over childhood. Obesity 16: 2663-2668.

34. Stovitz SD, Demerath EW, Hannan PJ, Lytle LA, Himes JH, 2011 Growing into obesity: patterns of height growth in those who become normal weight, overweight, or obese as young adults. Am J Hum Biol 23: 635-641.

35. Andersson EA, Pilgaard K, Pisinger C, et al, 2010 Do gene variants influencing adult adiposity affect birth weight? a population-based study of 24 loci in 4,744 Danish individuals. PLoS ONE 5: e14190.

36. López-Bermejo A, Petry CJ, Díaz M, et al, 2008 The association between the FTO gene and fat mass in humans develops by the postnatal age of two weeks. J Clin Endocrinol Metab 93: 1501-1505.

37. Elks CE, Loos RJF, Sharp SJ, et al, 2010 Genetic markers of adult obesity risk are associated with greater early infancy weight gain and growth. PLoS Med 7: e1000284.

38. Daniels SR, 2011 Lipid concentrations in children and adolescents: it is not all about obesity. Am J Clin Nutr 94: 699-700.

39. O'Rahilly S, Farooqi IS, 2008 Human obesity: a heritable neurobehavioral disorder that is highly sensitive to environmental conditions. Diabetes 57: 2905-2910.

40. Xi B, Wang C, Wu L, et al, 2011 Influence of physical inactivity on associations between single nucleotide polymorphisms and genetic predisposition to childhood obesity. Am J Epidemiol 173: 1256-1262.

41. Vimaleswaran KS, Li S, Zhao JH, et al, 2009 Physical activity attenuates the body mass index-increasing influence of genetic variation in the FTO gene. Am J Clin Nutr 90: 425-428.

42. Ortega-Azorín C, Sorlí JV, Asensio EM, et al, 2012 Associations of the FTO rs9939609 and the MC4R rs17782313 polymorphisms with type 2 diabetes are modulated by diet, being higher when adherence to the Mediterranean diet pattern is low. Cardiovasc Diabetol 11: 137 . 